\title{
Influence of social exposure on eating-related heart rate changes
}

\author{
Jian Zeng ${ }^{1}$, Wanhui Wen ${ }^{1 \star}$, Mingui Sun ${ }^{2}$, Wenyan Jia $^{2}$ \\ ${ }^{1}$ College of Electronic and Information Engineering, Southwest University, Chongqing, China, \\ 400715 \\ ${ }^{2}$ Department of neurological surgery, University of Pittsburgh, Pittsburgh, PA, USA, 15213 \\ 3zengjian999@swu.edu.cn,cwenwanh@swu.edu.cn,drsun@pitt.edu, jiawenyan@gmail.com
}

Keywords: eating detection, social exposure, heart rate

\begin{abstract}
Eating influences the sympathetic nerve control of the heart. However, few work has been done to reveal the influence of social exposure on eating-related sympathetic nerve activity. This work analyzes 14 subjects' eating RR interval series acquired before, during and after their eating alone and eating with social exposure. Statistic test shows that social exposure has significant influence on eating-related RR interval change. Two RR interval parameters, the mean and the number of times of continuous RR decrease in more than 6 heart beats, are used to classify the eating data and the non-eating data. The true positive rate and the true negative rate of eating detection are respectively $82.14 \%$ and $78.57 \%$ for the leave-one-out cross validation of a SVM classifier.
\end{abstract}

\section{Introduction}

Eating detection is a key part of diet analysis which can reveal the healthy or unhealthy way of lifestyle. Many modalities, such as swallowing sound [1,2], chewing sound [3], food intake gestures [4], gastric activity [5] and cardiac responses [6,7], are used for eating detection [8]. These eating detection methods have their respective profits and constraints [5]. Among them, previous research shows that food intake has marked effect on the size and shape of the T-waves of the Electrocardiogram (ECG) [6], accelerate the heart rate and decrease many parameters of heart rate variability [7].

Due to the social character of people, eating can be alone or with social exposure. However, few research has been done to analyze the influence of social exposure on eating related heart rate changes. Therefore, the current work analyzes the influence of eating on heart rate, explores whether social exposure has significant influence on the heart rate during and after eating and obtains eating-specific heart rate features to detect eating.

\section{Data acquisition experiment}

Sixteen subjects are randomly selected to take part in the eating experiment. The experiment consists of two parts, eating alone and eating with social exposure. In each part of the experiment, there are about 7 minutes baseline data acquisition before eating and about 7 minutes recovery data acquisition after eating. Eating alone means that the subject sits at a chair in a lab and eats alone, and eating with social exposure means that three people silently sit toward the subject when he/she is eating. The experiment is performed at the lunch time or at the dinner time, and the duration of the eating varies among subjects, from about 14 minutes to 20 minutes. Before the experiment, the subjects sign the informed consent and are told about the procedure of the experiment. ECG data are acquired through Biopac MP150 wireless ECG module.

\section{Data analysis}

The ECG data of one subject has too many lost R waves, and there are many abnormally long 
RR intervals in another subject's RR interval series. Therefore, the data of these two subjects are excluded, and the data of the other 14 subjects are used for further analysis.

As the data before, during and after eating have different durations, they are cut to the same 400-second length. Then, the RR interval series are calculated by R peak locating in the ECG data. Before statistic analysis, the occasionally appeared abnormal RR intervals caused by the loss of $\mathrm{R}$ wave during data acquisition are eliminated from the RR interval series.

The boxplot of RR intervals of each experiment is shown in Figure 1. Two-sample $t$ test has shown that each subject's mean RR intervals during eating is significantly shorter than that before eating ( $p<0.001)$, no matter there is social exposure or not. Except subject 4 after eating with social exposure and subject 11 after eating without social exposure, the mean RR interval after eating is significantly shorter than that before eating $(p<0.002)$. Except subject 2 and subject 7 , the mean RR interval of each of the other 12 subjects during eating without social exposure is significantly different from that during eating with social exposure ( $p<0.05$ for subject 1 and $p<0.001$ for the other 11 subjects). Eight subjects have significantly longer mean RR intervals during eating with social exposure than that during eating without social exposure, and 4 subjects have significantly shorter mean RR intervals during eating with social exposure than that during eating without social exposure. Except subject 8, the mean RR interval of each of the other 13 subjects after eating without social exposure is significantly different from that after eating with social exposure $(p<0.005)$. Eight subjects have significantly longer mean RR intervals after eating with social exposure than that after eating without social exposure, and 5 subjects have significantly shorter mean RR intervals after eating with social exposure than that after eating without social exposure. In order to eliminate the influence of baseline difference, the RR intervals of eating with social exposure are compared with those of eating alone after they subtract the baseline mean RR interval acquired before eating.

As there is a consistent trend of mean RR decrease caused by eating of all the subjects, we have focused on the RR decreasing process of the RR interval series and observed continuous RR decrease in more than 6 heart beats which has rarely appeared before eating, e.g. the RR interval series of subject 13 shown in Figure 2. The number of times of continuous RR decrease in more than 6 heart beats, $N_{\mathrm{d}}$, is shown in Figure 3. For individual subject, $N_{\mathrm{d}}$ during eating is larger than that before eating. $N_{\mathrm{d}}$ after eating is smaller than that during eating, except those of subject 6 and subject 7. That is to say, for some people, there would be an obvious delay between eating and its influence on the sympathetic nerve system.

As the mean RR interval and the $N_{\mathrm{d}}$ show consistent variation trend within individual subject, they are used to describe the before eating and during eating datasets into 56 2-dimensional data vectors, and a SVM classifier is applied to distinguish the eating data from the non-eating data. The true positive rate and the true negative rate are respectively $82.14 \%$ and $78.57 \%$ for the leave-one-out cross validation of a SVM classifier. 


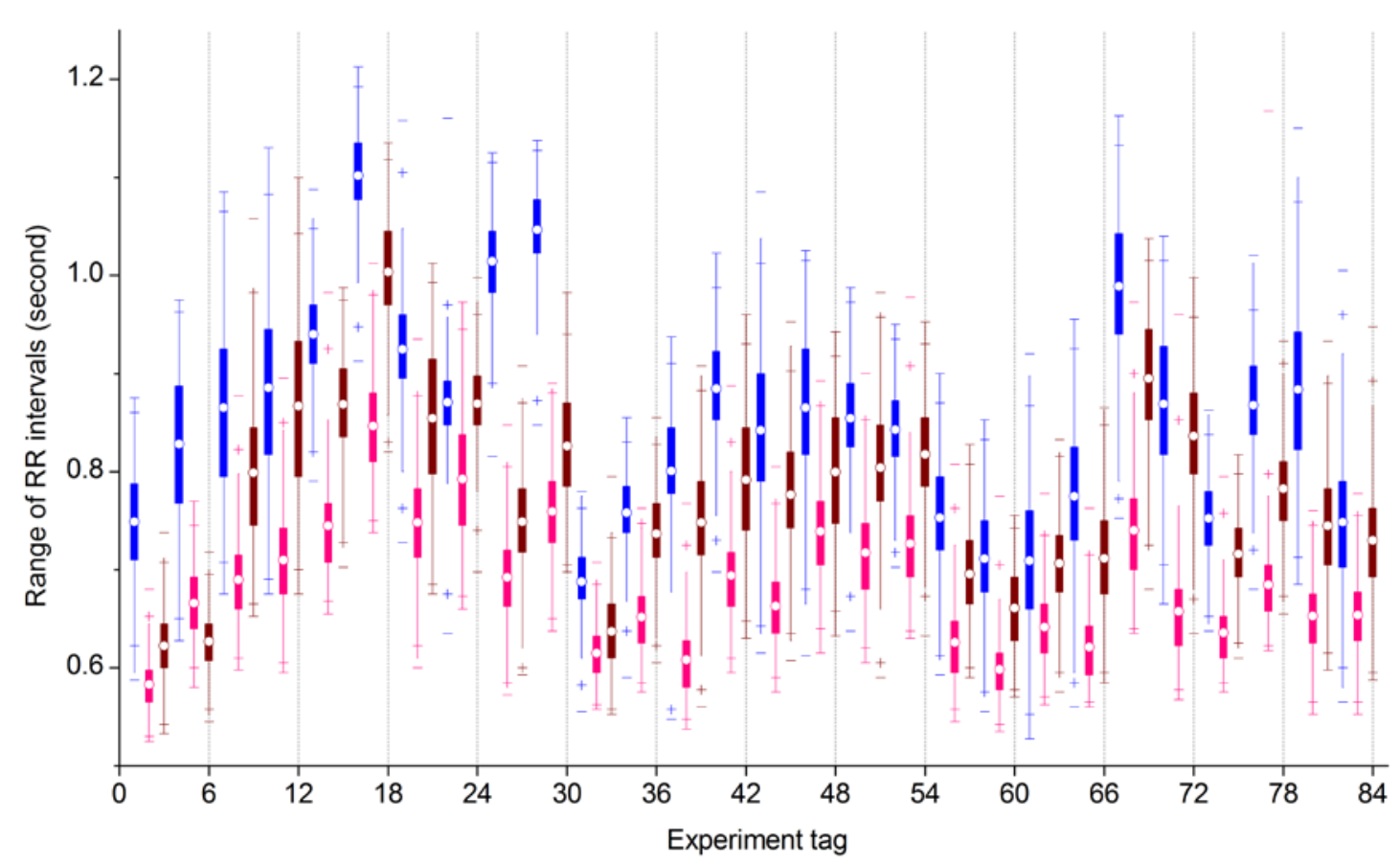

Fig. 1 The range of RR intervals of each eating experiment. The data belonging to one subject are

put in the same grid. For example, the six bars shown in grid 1 belong to subject 1 and are respectively the range of RR intervals before, during and after eating without social exposure and before, during and after eating with social exposure.

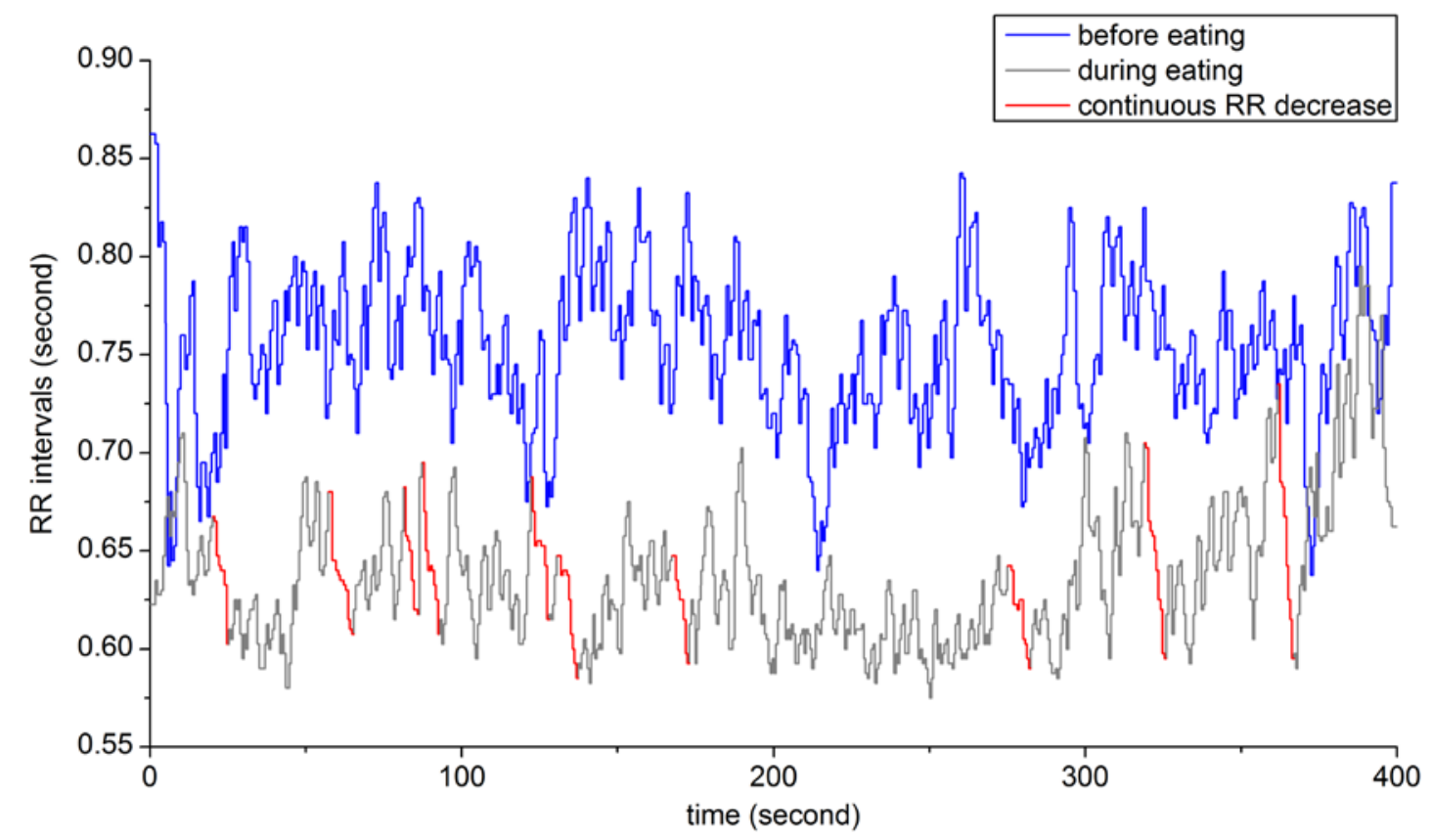

Fig. 2An example of RR interval series before and during eating. Specifically, the RR intervals during eating show the character of continuous RR decrease in more than 6 heart beats. Although there might be once of RR increase or equal RR intervals during the process of continuous RR decrease, the trend of such continuous RR decrease seems to be eating-specific. 


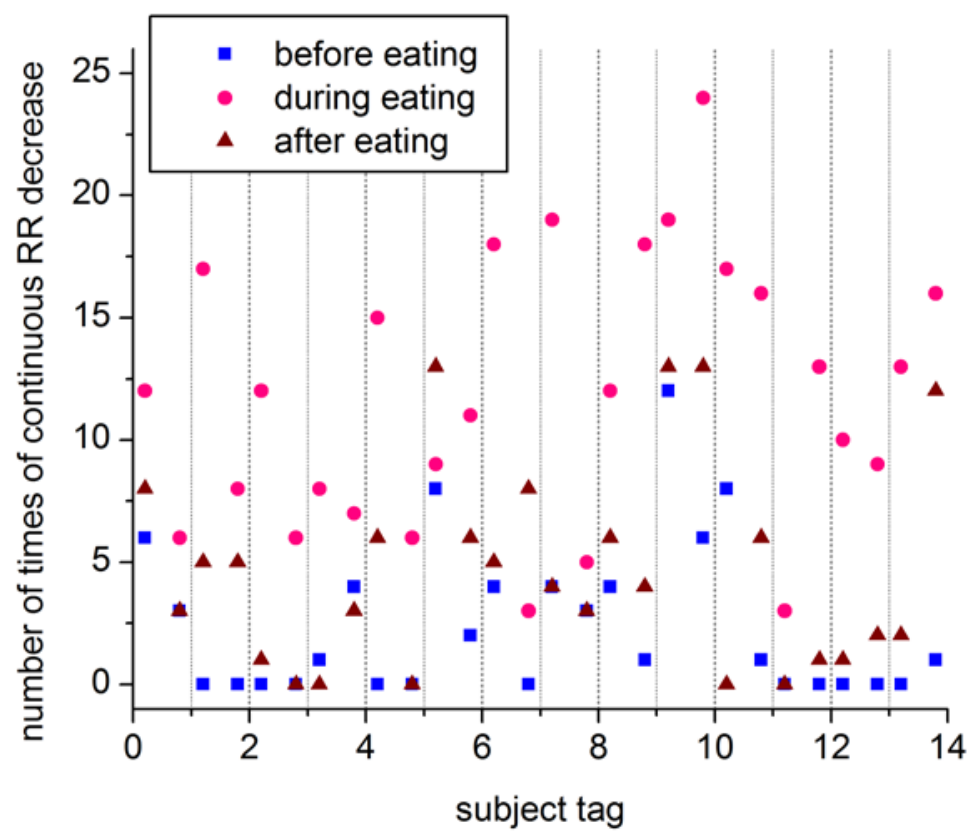

Fig. 3The number of times of continuous RR decrease in more than 6 heart beats. The data belonging to one subject are put in the same grid.

\section{Discussion}

The $t$ test results reveal that eating leads to the acceleration of the heart rate which is the result of the activated sympathetic nerve control. For most subjects, the activated sympathetic nerve system does not calm down immediately after eating, and the effect of eating on the sympathetic nerve system is equivalent to a kind of moderate physical stress. Therefore, the heart rate during eating should not be too slow or too fast. In the above-mentioned eating experiment, the mean heart rate during eating is in the range of $70 \mathrm{BPM}$ to $103 \mathrm{BPM}$.

Social exposure has significant influence on the heart rate of most subjects during and after eating. However, such influence can be different among the subjects, and more subjects have shown relieved physical stress when they were eating with social exposure.

Although there are individual differences among the subjects, the mean RR interval and the number of times of continuous RR decrease in more than 6 heart beats appear eating-specific trend within individual subject, and the SVM classifier has obtained much better eating detection result than random guess. Therefore, these two parameters are promising features for eating detection.

\section{Acknowledgment}

This work is supported by National Science Foundation of China (Grant No. 61103132 and No. 61472330), the Fundamental Research Funds for the Central Universities (Grant No. XDJK2013A020) and National Institutes of Health (Grant No. R01CA165255) in U.S.A.

\section{Reference}

[1] T.Olubanjo and M.Ghovanloo. Real-time swallowing detection based on tracheal acoustics. In proceeding of Acoustics, Speech and Signal Processing (ICASSP), 2014 IEEE International Conference on, 2014, pp. 4384 - 4388. DOI: 10.1109/ICASSP.2014.6854430.

[2] M.Farooq, J.M.Fontana and E.Sazonov. A novel approach for food intake detection using electroglottography. Physiological measurement, 2014, 35: 739-751. DOI: 10.1088/0967-3334/35/ 5/739.

[3] E.Sazonov and J.M.Fontana. A sensor system for automatic detection of food intake through non-invasive monitoring of chewing. IEEE Sensor Journal, 2012, 12(5): 1340-1348. DOI: 
10.1109/J SEN.2011.2172411.

[4] Y.Bai, W.Jia, Z.Mao and M.Sun. Automatic eating detection using a proximity sensor. In proceeding of Bioengineering Conference (NEBEC), 2014 40th Annual Northeast, 2014, pp. 1-2. DOI: 10.1109/NEBEC.2014.6972716.

[5] O.Amft and G.Troster. On-body sensing solutions for automatic dietary monitoring. IEEE Pervasive Computing, 2009, 8 (2): 62-70. DOI: 10.1109/MPRV.2009.32.

[6] E.Widerlov, K.G.Jostell, L.Claesson, B.Odlind, M.Keisu and U.Freyschuss. Influence of food intake on electrocardiograms of healthy male volunteers. European Journal of Clinical Pharmacology, 1999, 55 (9): 619-624.

[7] S.Passler, A.Noack, R.Poll and W.J.Fischer. Validation of the use of heart rate variability measurements during meal intake in humans. In proceeding of Computing in Cardiology Conference (CinC), 2013, pp. 999-1002.

[8] J.M.Fontana, M.Farooq and E.Sazonov. Automatic ingestion monitor: a novel wearable device for monitoring of ingestive behavior. IEEE Transactions on Biomedical Engineering, 2014, 61 (6): 1772-1779. DOI:10.1109/TBME.2014.2306773. 